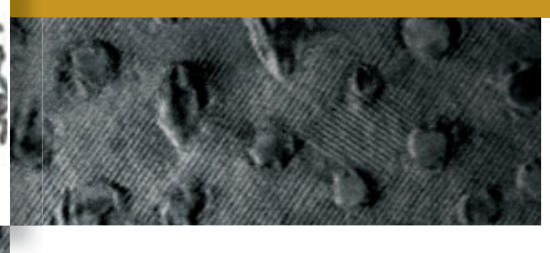

\title{
Obtaining ultimalte functionalities in nanocomposites: Design, control, and fabrication
}

\section{Ce-Wen Nan and Quanxi Jia, Guest Editors}

\begin{abstract}
Emergent behavior can be achieved in composites by interfacing different materials at the nano- or mesoscales. Integrating different materials on a single platform or forming composite provides a new design paradigm to yield enhanced or novel functionalities that cannot be obtained in individual constituents. Nanocomposites, in particular, have been model systems for enhancing interface effects on physical properties because they provide reduced dimensionality or enlarged interfacial areas. To fabricate technologically relevant multifunctional materials, one needs to understand and control the interactions in different materials by manipulating interfaces at the nano- or mesoscales. This issue of MRS Bulletin focuses on nanocomposites, with an emphasis on approaches to the design and control of the functionalities of composite materials through controlled synthesis and advanced characterization in concert with simulation and modeling.
\end{abstract}

\section{Introduction}

Composites are a class of materials that combine two or more constituents into a form suitable for technological applications. Although each constituent retains its chemical and structural identity, the composite might display macroscopic multifunctionalities, superior to those of its parent constituents, or completely new functionalities. A classic example is fiberglass-reinforced plastics (FRPs), developed during the 1940s. ${ }^{1}$ The combination of glass fibers with a thermoset resin matrix launched a commodity industry that currently provides products in a diversity of market areas. Identifying the right combination of two or more known substances especially on the nanoscale has become an effective method for designing and developing entirely new materials with unique and desired properties not obtainable in existing materials. The search for environmentally friendly and application-specific smart materials using nanostructured composites, or nanocomposites, is a much-pursued effort.

Nanocomposites, in which at least one of the phases has a dimension in the range of a few angstroms to tens of nanometers, have been recognized as one of the most appropriate materials systems for producing multifunctional properties. Indeed, they represent an engineering solution to synergistically integrate the properties of different materials in a single platform. Examples include polymer-based, metal-based, and oxide-based nanocomposites, as well as organic-inorganic hybrid materials. ${ }^{2}$
In nanocomposites, emergent behavior can be achieved by interfacing different materials at the nano- or mesoscales. In other words, the properties of a composite can be greatly enhanced in comparison with those of individual constituents. Even more appealing, novel and emergent properties that are not exhibited by any of the constituents in the composite can be produced through synergistic coupling interactions. The synergistic integration of known materials to form composites provides new opportunities and strategies for fabricating smart materials with desired properties for targeted applications.

\section{Microstructures}

The macroscopic properties of nanocomposites depend strongly on the properties of their constituents and their microstructures. The detailed spatial arrangements of these constituents also play an important role in determining the ultimate functionalities of the nanocomposites. Although the properties of the constituents are often assumed to be known, the microstructures and boundary conditions between the two phases can largely control the properties of the resulting nanocomposites. Optimizing microstructures and interfaces is especially important in achieving desired device performance for specific applications (see the article in this issue by $\mathrm{Hu}$ et al.). ${ }^{3}$

Figure 1 shows the most commonly studied architectures of nanocomposites. The connectivity of each phase in the composite 

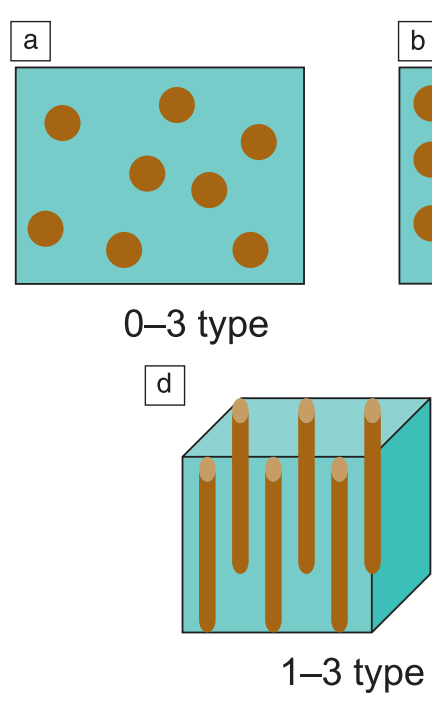
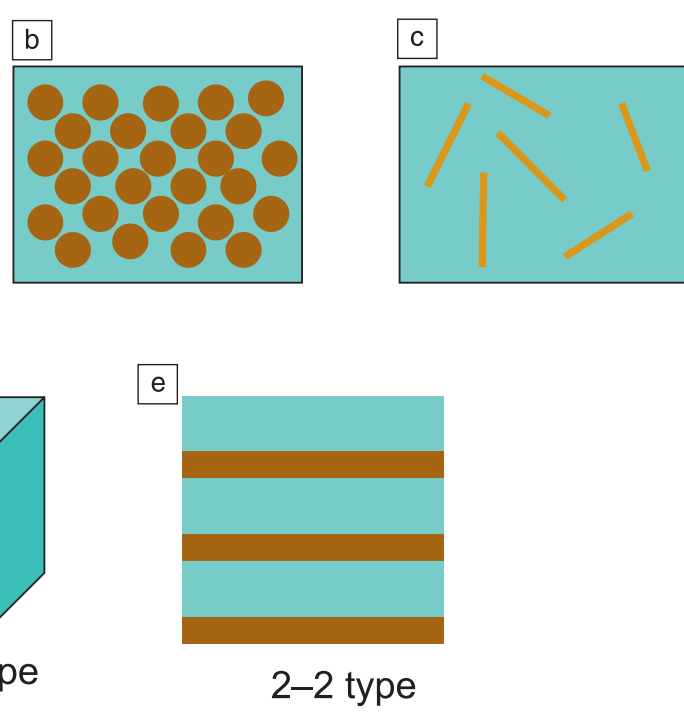

Figure 1. Schematic illustrations of the most commonly investigated nanocomposite architectures: (a) 0-3-type nanocomposite with nanoparticles dispersed in a matrix; (b) periodic nanoparticle arrangement obtained by self-assembly, for example; (c) 0-3-type nanocomposite with nanofibers (nanowires, nanotubes) or nanoplates dispersed in a matrix; (d) 1-3-type nanocomposite with nanofibers or nanopillars aligned in a matrix; and (e) 2-2-type nanolaminate or heterostructured thin films or superlattices.

throughout the system can be achieved even if the volume fraction is at a very low level (as low as $\left.10^{-3}\right) \cdot{ }^{6,7}$ In addition, the nanofibers can be oriented to form nanocomposites with anisotropic properties. One example is the formation of a well-aligned CNT forest embedded in a superconducting $\mathrm{NbC}$ matrix. The CNTs function as effective vortex pinning centers that allow the $\mathrm{NbC}$ to maintain high current-carrying capability at high magnetic fields. The CNT-NbC composite shows much enhanced and anisotropic upper critical and irreversibility fields. ${ }^{8}$

Two well-known microstructures that exhibit anisotropic properties are the so-called 1-3 and 2-2 types, schematically illustrated in Figure 1d-e, respectively. The 1-3-type nanocomposite is usually composed of continuous nanofibers that are aligned along a certain direction in the matrix. The morphology of the epitaxial nanocomposite films is mostly controlled by the surface and interfacial energy, deposition temperature, growth rate, volume ratio of the two phases, and lattice match

(matrix and second phase) can be in zero, one, two, or three dimensions, as indicated by the notation. It should be noted that the detailed microstructure of an individual phase can vary, depending on the application. For instance, the nanoparticle itself can have a core-shell structure (see the articles in this issue by Hollingsworth et al. and Shen et al.). The stacking unit can be $[\mathrm{A} / \mathrm{B}]_{N}$ or $[\mathrm{A} / \mathrm{B} / \mathrm{C}]_{N}$, where $\mathrm{A}, \mathrm{B}$, and $\mathrm{C}$ are different single-phase materials, and $N$ is the stacking periodicity of the nanostructure.

Shown in Figure 1a is the so-called 0-3-type composite, in which nanoparticles are randomly dispersed in a matrix. One example is the inclusion of $\mathrm{BaZrO}_{3}$ nanoparticles in hightemperature superconducting $\mathrm{YBa}_{2} \mathrm{Cu}_{3} \mathrm{O}_{7}$ films to enhance the current-carrying capability under a magnetic field ${ }^{4}$ (also see the article in this issue by Zhang et al.). When the volume of dispersed nanoparticles is increased, the nanoparticles can be regularly dispersed in the matrix (Figure 1b) by certain in situ growth processes such as self-assembly. In this case, a relatively homogeneous microstructure with a periodic dispersion of particles can be formed. Photonic crystals ${ }^{5}$ are a typical example with a porous periodic microstructure formed by self-assembly.

When a small amount of nanofibers (e.g., carbon nanotubes [CNTs]) or nanoplates (platelet-shaped nanoparticles [e.g., graphene, nanoclays]) is dispersed in the matrix, a similar 0-3-type microstructure (Figure 1c) can also be formed. Numerous nanocomposites with included CNTs or graphene have been developed to provide unique properties, taking advantage of the large aspect ratios of the nanofibers. For example, nanofibers can readily touch each other even at low volume fractions, so that a continuous percolation of clusters between the substrate and the individual phases (see the article in this issue by Zhang et al.). 2-2-type nanocomposites are usually used to label nanolaminated layered structures and even superlattices.

\section{Interfaces}

The functionalities of nanocomposites are closely related not only to the intrinsic properties of the individual phases, but also to the boundary conditions across the heterointerfaces of different phases. In other words, the large surface areas and nanometer scale of the individual phases need to be taken into consideration in the design of nanocomposites for specific applications. As illustrated in Figure 2a, in polymer-based nanocomposites, the interface divides the inorganic and organic phases into two distinct regions. A transition region across the interface can exist if the inorganic nanoparticle is functionalized (see the enlarged view in the illustration of the nanoparticle). The inorganic nanoparticles are embedded in the organic matrix, and only weak bonds (hydrogen or van der Waals) are formed across the heterointerfaces.

The design of nanoparticle-polymer nanocomposites requires the rational selection of both the nanoparticles and the polymer matrix, where their interaction and consequent effects on the dispersion of nanoparticles, the nano- and mesoscale morphologies of the polymer matrix, and the interface between the inorganic particle and organic matrix are taken into consideration (see the article in this issue by Shen et al.). In inorganicinorganic nanocomposites, the interface can be quite different in nature because the two phases are linked together by strong chemical bonds (covalent or ionic-covalent bonds). The detailed microstructures across the heterointerfaces depend on the lattice 


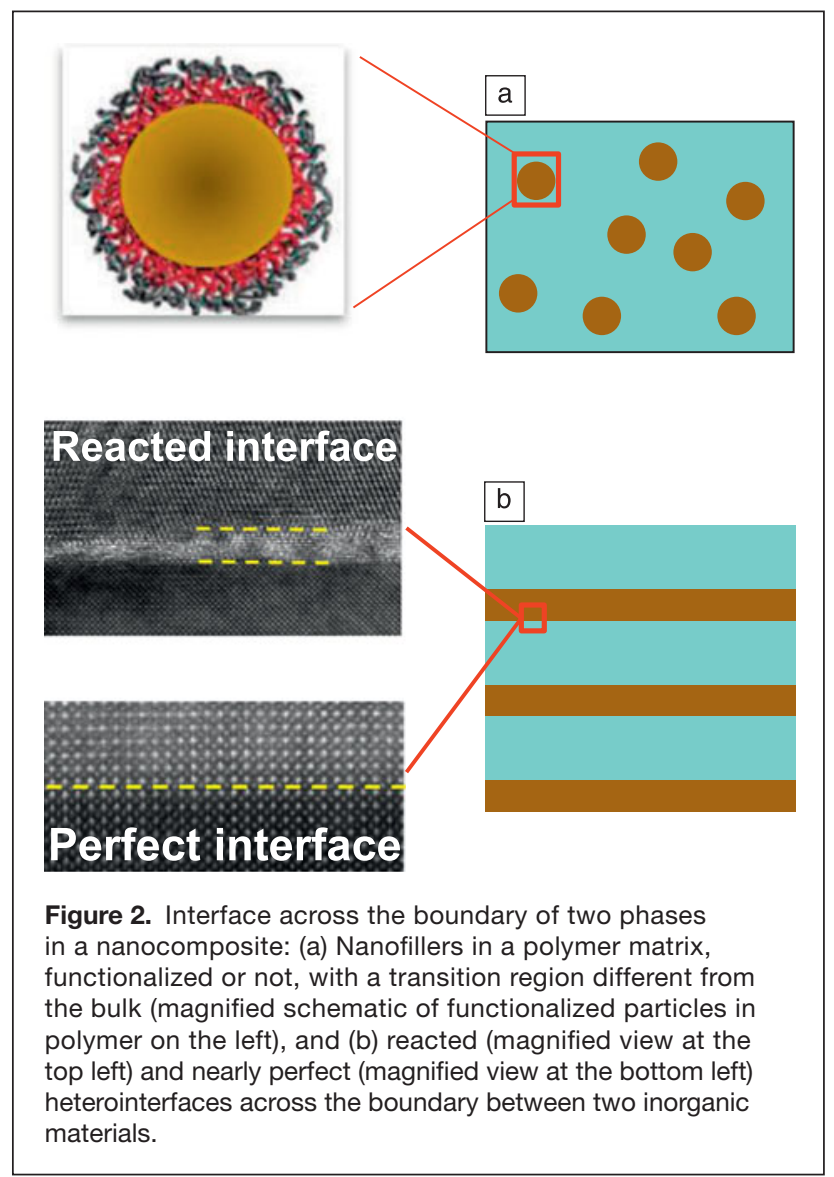

mismatch and the processing conditions used (see the magnified transmission electron microscopy images in Figure 2b).

The interfacial region can be as thin as several angstroms to as thick as a few nanometers. Although the interfacial transition region is very thin, its properties are usually different from those of the constituents. The transport properties of the interfacial region can be even more complex if interdiffusion takes place. In practice, the interface is usually not perfect because of interface bond mismatch and defects, thermal expansion mismatch and thermal stress, and chemical reaction of the constituents at or near the interface. Interfaces can have multiple effects on phonon transport (see the article in this issue by Liao and Chen) and can be very complex, both of which are core issues in the control of functionalities of nanocomposites.

Imperfect interfaces often lead to discontinuities in local fields across the interface. For transport properties, there are two distinct types of interfaces. A Type I interface is characterized by a discontinuity of the potential function $\Psi$ at the interface $S_{12}$ (see Figure 3a, where $S_{12}$ denotes the interface between phases 1 and 2 , and $\delta$ is the thickness of the interface).
This kind of interface always acts as a barrier layer, as seen with widely investigated nanocomposites with CNTs or graphene as fillers. The Type II interface is characterized by a discontinuity in the carrier flow density $Q$ at the interface (Figure $3 b$ ). In this case, the carriers flow along the interface, and the interface acts like a "superconducting" migration channel., 10

\section{Why nanocomposites?}

The technological relevance of nanocomposites lies in the emergent behavior achieved by interfacing various known materials at the nano- or mesoscales. Furthermore, there is more freedom to tune or enhance functionalities that cannot be obtained from individual constituents. The microstructure and thus the macroscopic properties of nanocomposites can be designed as required. Combinations of known materials with appropriate architectures at the nano- or mesoscales provide a rational approach to the design of materials for specific applications.

A nanocomposite is not simply a structural variation at the nano- or mesoscales. The synergic interaction between the individual constituents plays an important role in enhancing or improving the functionalities, as well as producing emergent phenomena. There are two intriguing composite effects (see Figure 4). ${ }^{11}$ For the $1+1>2$ composite effect, the properties of the composites can be greatly enhanced or become unbounded over those of the constituents (Figure 4a). For the $0+0>0$ composite effect, completely new properties (see a phenomenological demonstration in Figure $4 b$ ) that do not exist for any of the constituents can be produced in the nanocomposites.

One example of the $1+1>2$ composite effect is the dielectric nanocomposite, with conductive nanoparticles dispersed in a dielectric polymer. The dielectric constant of such a nanocomposite diverges at the percolation threshold $f_{\mathrm{c}}$, at which

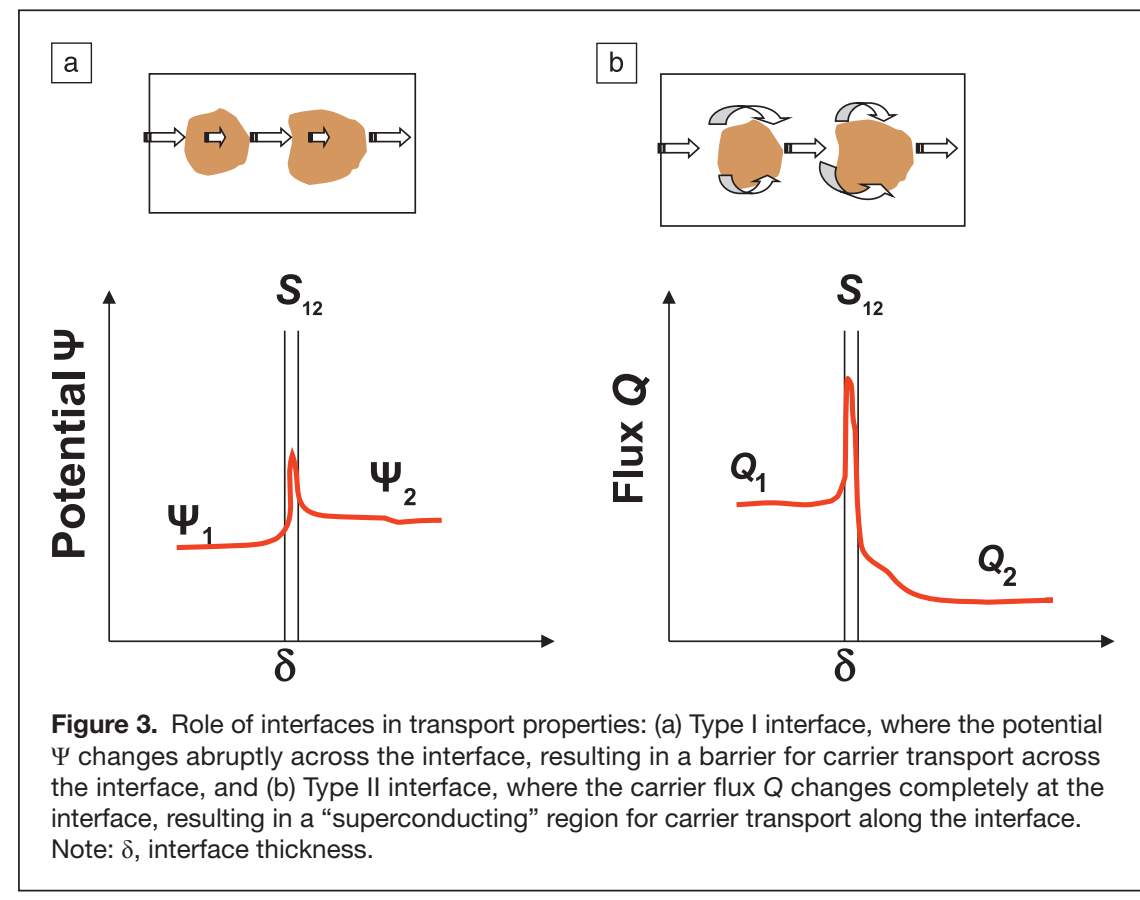



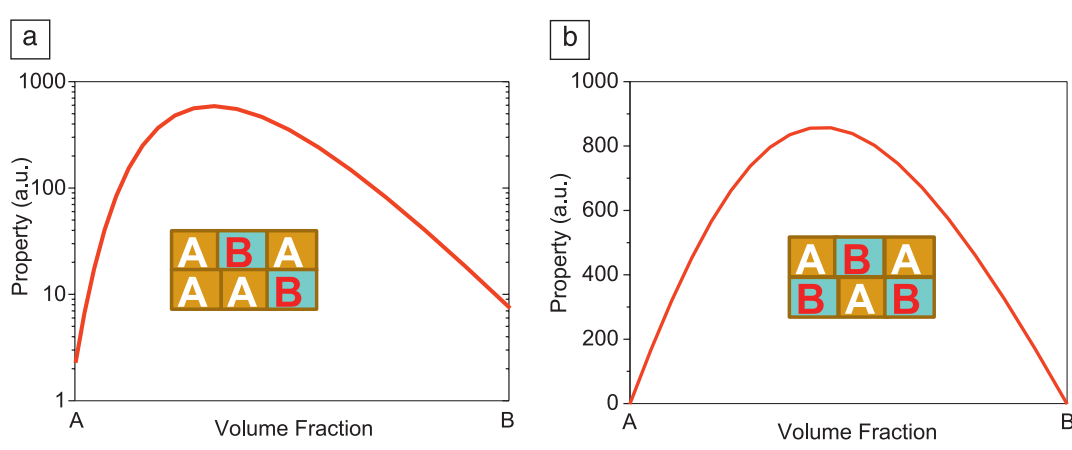

Figure 4. Schematic illustration of composite effects obtained by rational combinations of constituents $A$ and $B$ : (a) $1+1>2$ composite effect, where enhanced or improved properties of $A$ and $B$ are obtained, and (b) $0+0>0$ composite effect, where emergent behavior, not exhibited by individual constituents $A$ and $B$, is achieved. Adapted from Reference 11.

ultimate functionalities of composite materials through controlled synthesis and advanced characterization in concert with simulation and modeling.

Multiferroic magnetoeletric nanocomposites, which simultaneously exhibit ferroelectricity and ferromagnetism at the nanoscale, have stimulated intense research activities over the past decade because of their scientific appeal as interesting materials with great potential applications. $\mathrm{Hu}$ et al. review multiferroic magnetoeletric nanocomposites and discuss the approaches and challenges that need to be addressed in pursuing practical applications of magnetoelectric devices.

Lattice-strained epitaxial nanocomposite films provide a new design paradigm to tune

sufficient nanoparticles are present to form a long-range connected network. ${ }^{12}$ Many experiments have shown that, near to $f_{\mathrm{c}}$, the dielectric constant of the composite can become unbounded and extraordinarily high compared to that of the dielectric matrix. This composite effect comes from many conductive nanoparticles being isolated by thin dielectric layers, thus forming nanocapacitors. In general, a large $1+1>2$ composite effect can be expected by carefully choosing the constituents and designing the geometry of their combinations. For instance, Solin and coworkers ${ }^{13}$ observed extremely high room-temperature magnetoresistance in a simple composite nanostructure of $\mathrm{Au}$ and InSb. Such greatly enhanced magnetoresistance is due to current deflection by the Au inhomogeneity, which acts as a short circuit at zero magnetic field but as an open circuit at high fields. In nanoparticle-polymer nanocomposites, nanoparticle assembly within a polymer can lead not only to enhancements or changes in the properties of nanoparticles, but also to significant change in the intrinsic properties of the polymer (see the article in this issue by Firestone et al.).

The $0+0>0$ composite effect offers a unique way to explore new classes of multifunctionalities with known ordinary materials. One approach to producing the $0+0>0$ composite effect is through the elastic coupling interaction of the constituents. An attractive example is the novel magnetoelectricity produced in multiferroic composites by integrating magnetic and ferroelectric materials, neither of which exhibit a magnetoelectric effect (see the article in this issue by $\mathrm{Hu}$ et al.).

Many attempts have been made to pursue $1+1>2$ or $0+0>0$ composite effects. The future lies in better understanding the relationships between the structures and the composite effects. The composite effects have not yet been fully explored, and the possibilities are limited only by the imagination.

\section{In this issue}

The six articles in this issue cover a range of topical areas with an emphasis on approaches to designing and controlling the and manipulate functionalities that cannot be obtained in individual constituents. Tuning and controlling the functionalities of a broad range of materials utilizing such a scheme is emerging as an exciting direction in materials research. Zhang et al. provide an overview of multifunctional self-assembled oxide nanocomposite thin films and devices. Examples are given to illustrate that the ability to synthesize self-assembled nanocomposite thin films of complex metal oxides has provided pathways to manipulate interfaces and thus achieve novel physical phenomena.

Nanocomposites have shown potential thermoelectric applications, although "nano" might not be the best approach for decreasing thermal conductivity because of the increased interfacial resistance in such materials. Nevertheless, it is clear that an ideal thermoelectric material should possess a combination of properties of nondegenerate semiconductors, metals, insulators, and amorphous materials to enhance the nondimensional figure of merit, and composites would therefore be a promising route toward better thermoelectrics. Liao and Chen review emerging principles of heat and charge transport in nanocomposites and discuss working examples of thermoelectrics, as well as thermal engineering in general.

Organic-inorganic nanocomposites could find wide applications in energy storage, electrocatalysis, display, photovoltaics, and sensors, to list a few. Challenges, however, remain because the interface between the organic and inorganic materials and the coupling effect between these two phases are not well understood. Shen et al. review polymer nanocomposites, in which high-dielectric-constant (high- $k$ ) nanofillers are incorporated in a polymer matrix. This overview clearly shows that innovative strategies need to be developed to balance the seemingly contradictory demands of enhancing dielectric constants while maintaining high dielectric strength and to fully explore the promise and potential of nanocomposites.

The ongoing pursuit of multifunctional soft materials can impact a wide range of technological challenges ranging from information processing to energy storage and transducing devices. The complex interplay of properties that are intimately 
mixed at the nanoscale can lead to useful and unexpected behaviors that are not present in either component. Firestone et al. highlight recent literature examples of composites in which nanoparticle assembly within polymers leads to enhancement or changes in the nanoparticle properties. They also discuss how introducing nanoparticles into a polymer can cause significant changes in the intrinsic properties of the polymer.

Colloidal semiconductor nanocrystals or quantum dots have found potential applications as light-emitting devices and thin-film displays. Significant progress has been made over the past decade in the self-assembly of colloidal nanoparticles. However, efforts to exploit new functionality resulting from ordered arrays of dissimilar but interacting nanoparticles remains limited in the field. Hollingsworth et al. review techniques for creating hybridized functional composites of quantum-confined semiconductor nanostructures and plasmonic nanocrystals, and the effects of hybridization. They emphasize assembly approaches that have been used to create semiconductor-metal hybrids and, in particular, those that have afforded specific plasmonic effects on excitonic properties. They also review direct-synthesis and chemicallinker approaches to creating discrete semiconductor-metal interactions.

\section{Concluding remarks}

As the design and development of new materials becomes more costly and complex, the synergistic integration of known materials with desired properties to form nanocomposites becomes an increasingly attractive strategy for multiple functionalities and emergent behavior. It is hoped that the perspectives presented in this issue will encourage new thinking toward bringing scholars across multiple disciplines (materials science, chemistry, physics, engineering, computation) together.

A closed-loop effort involving in-depth understanding of the links between processing, microstructure, and properties, and control of microstructures of nanocomposites has played an important role in the successful development of many technologically relevant materials. To accelerate fundamental understanding and to obtain ultimate functionalities in nanocomposites, intimate coupling of synthesis and characterization with modeling and simulation at different length and time scales needs to be emphasized. Furthermore, advanced probing techniques need to be exploited to effectively analyze and observe buried interfaces and local structural and transport inhomogeneities that control the functionalities of a range of materials. To realize controlled and desired functionalities for specific applications, we need broad innovations at the boundaries of materials science, chemistry,

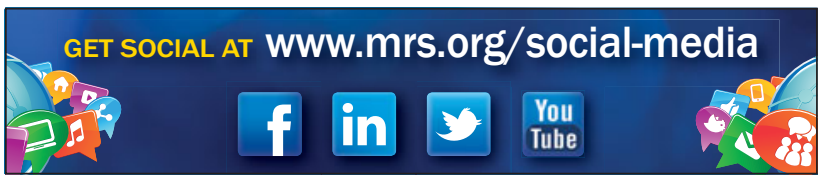

physics, theory, and engineering that can facilitate materials design and fabrication.

\section{Acknowledgments}

The work at Tsinghua University was supported by the NSF of China (Grant No. 51221291). The work at Los Alamos was supported by the Laboratory Directed Research and Development Program and the Center for Integrated Nanotechnologies, an Office of Science User Facility operated by the U.S. Department of Science.

\section{References}

1. M. Lavine, Science 314, 1099 (2006).

2. C. Sanchez, B. Julian, P. Belleville, M. Popall, J. Mater. Chem. 15, 3559 (2005).

3. R.E. Newnham, D.P. Skinner, L.E. Cross, Mater. Res. Bull. 13, 525 (1978).

4. J.L. MacManus-Driscoll, S.R. Foltyn, Q.X. Jia, H. Wang, A. Serquis, L. Civale, B. Maiorov, M.E. Hawley, M.P. Maley, D.E. Peterson, Nat. Mater. 3, 439 (2004).

5. S. John, Nat. Mater. 11, 997 (2012).

6. S. Stankovich, D.A. Dikin, G.H.B. Dommett, K.M. Kohlhaas, E.J. Zimney, E.A. Stach, R.D. Piner, S.T. Nguyen, R.S. Ruoff, Nature 442, 282 (2006).

7. M.B. Bryning, M.F. Islam, J.M. Kikkawa, A.G. Yodh, Adv. Mater. 17, 1186 (2005).

8. G. Zou, H. Luo, S. Baily, Y. Zhang, N. Haberkorn, J. Xiong, E. Bauer, T. McCleskey, A. Burrell, L. Civale, Y.T. Zhu, J.L. MacManus-Driscoll, Q.X. Jia, Nat. Commun. 2, 248 (2011).

9. N. Sata, K. Eberman, K. Eberl, J. Maier, Nature 408, 946 (2001)

10. A. Ohtomo, H.Y. Hwang, Nature 427, 423 (2004).

11. C.W. Nan, Z. Metallkde. 94, 1148 (2003).

12. C.W. Nan, Y. Shen, J. Ma, Annu. Rev. Mater. Res. 40, 131 (2010).

13. S.A. Solin, T. Thio, D.R. Hines, J.J. Heremans, Science 289, 1530 (2000). $\square$

\section{SPECTROSEOPY OF MICROSCOPIC SAMPIES}

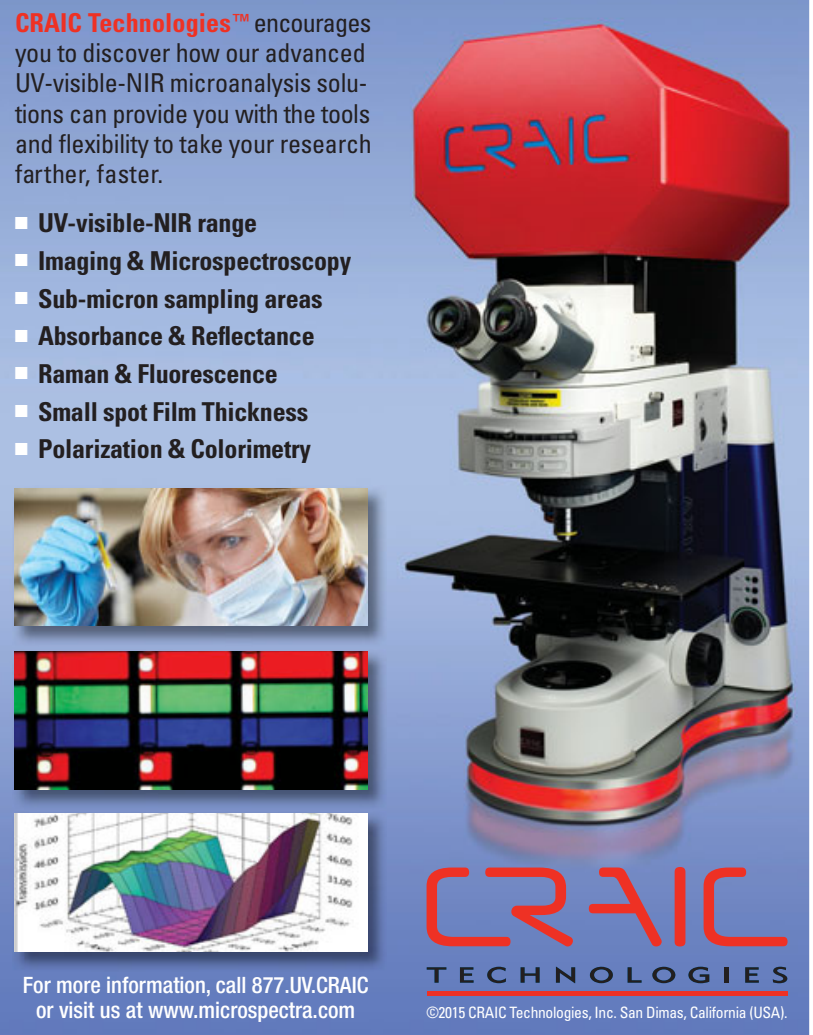
you to discover how our advanced UV-visible-NIR microanalysis solutions can provide you with the tools and flexibility to take your research farther, faster.

UV-visible-NIR range Imaging \& Microspectroscop Sub-micron sampling areas Absorbance \& Reflectance Raman \& Fluorescence Small spot Film Thickness Polarization \& Colorimetry 

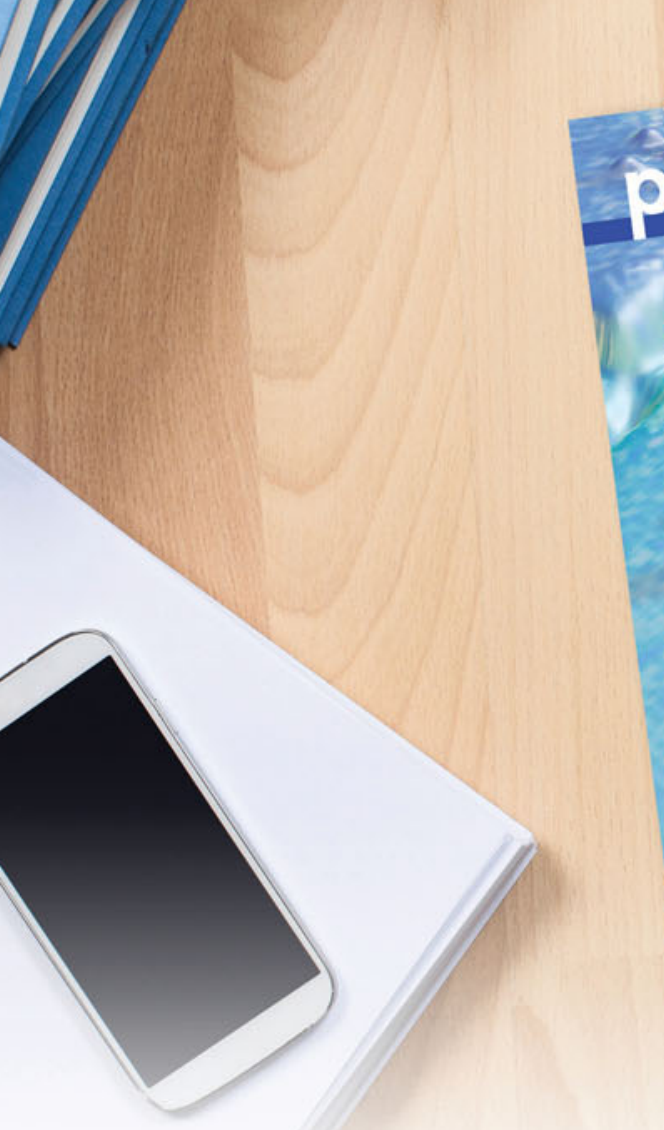

\section{Aspin down memory lane}

\section{Researchers who don't have time to read magazines, make time to read Physics Today.} Join the community of 120,000 readers across the physical sciences for just $\$ 25 /$ year.

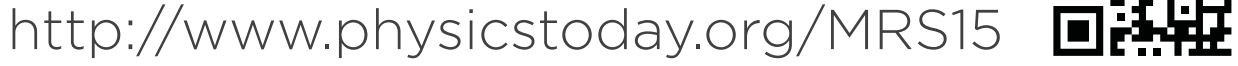

